\title{
Neurophysiological sensitivity for impaired phonological processing in the acute stage of aphasia
}

\author{
Annelies Aerts a , Pieter van Mierlo ${ }^{b}$, Robert J. Hartsuiker c , Patrick Santens ${ }^{\text {a,d }}$ and Miet De Letter d,e
}

${ }^{a}$ Dept. of Internal Medicine, Ghent University, ${ }^{b}$ Dept. of Electronics and Information Systems, Ghent University - Iminds, c Dept. of Experimental Psychology, Ghent University, ${ }^{d}$ Dept. of Neurology, Ghent

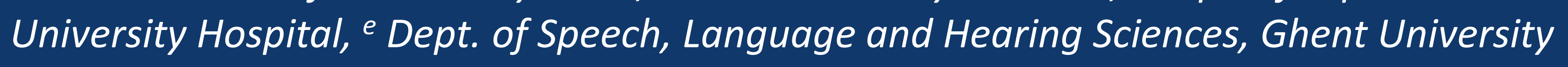

\section{BACKGROUND}

\section{Possible to investigate phonological input processes using event-related} potentials (ERPs)

Mismatch Negativity (MMN) and P300 reflect phoneme discrimination

$\rightarrow$ pre-attentive and attentive deviance detection in oddball task (interspersing frequent stimuli with rare, infrequent stimuli)

\section{Possible to investigate single-word processing using ERPs}

N400 for word recognition/comprehension

$\rightarrow \mathrm{N} 400$ to pseudowords (PW) larger than N400 to real words $(\mathrm{RW})=$ 'pseudoword effect'
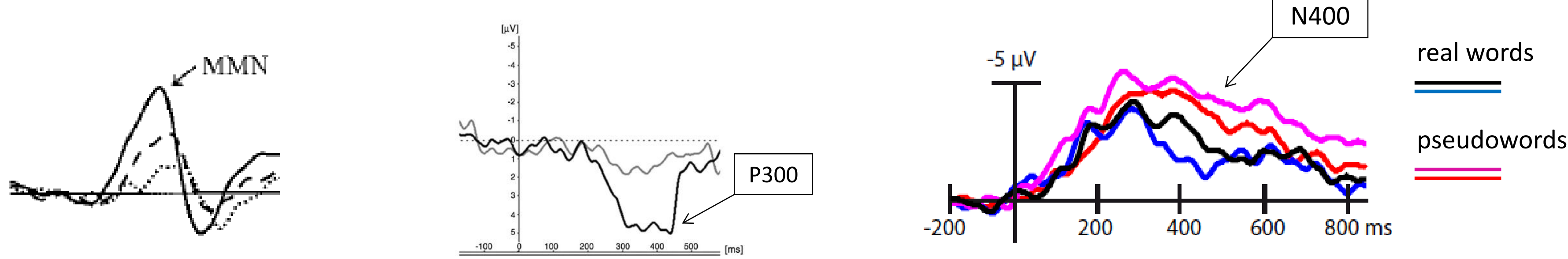

Studies implementing these ERPs in aphasic patients work with chronic patients and use non-speech stimuli or do not compare different speech stimuli (distinctive features):

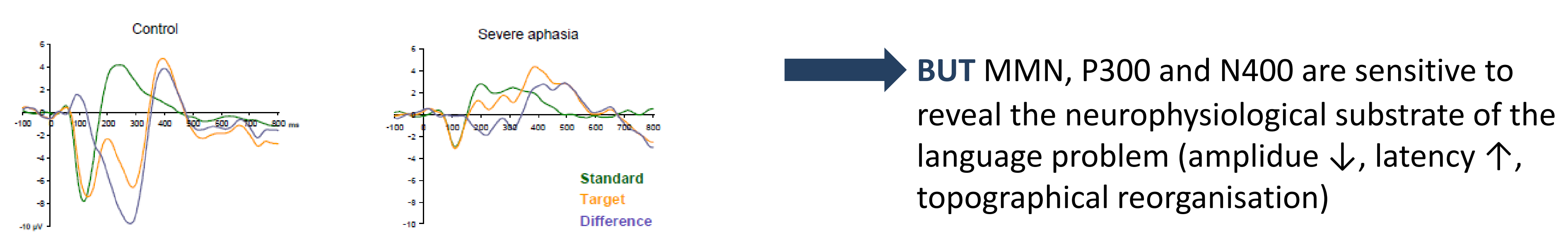

\section{Only a few studies have used ERPs in acute aphasic patients [1,2] !!!}

$\rightarrow$ NONETHELESS ERPs = valuable complement in the clinical evaluation of acute aphasic patients $\rightarrow$ when behavioural assessment is difficult or impossible!

$\rightarrow$ Before clinical use, the following research questions first need to be answered:

1) Difference between place, manner and voicing during pre-attentive and attentive auditory phoneme discrimination?

2) What is the effect when more attention is required? Is the attentive task appropriate for clinical use?

3) Can acute aphasic patients discriminate between real words and pseudowords?

\section{MATERIALS AND METHOD}

\section{Patient group}

10 patients: 5 men $/ 5$ women Mean age: 69.4 jaar (+/- 3.46)

$<2$ weeks post-stroke $=$ acute stage!

\section{Norm group ${ }^{[3]}$}

44 subjects: 20 men/24 womer

Equivalent mean age $(p=0.785)$

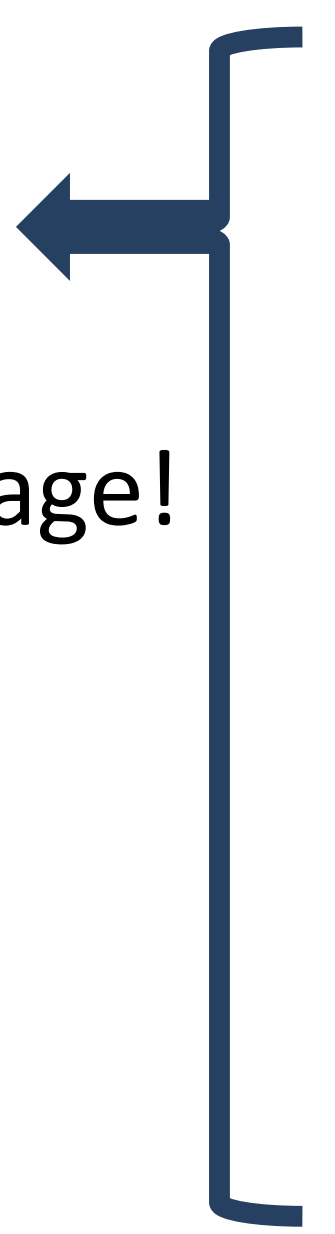
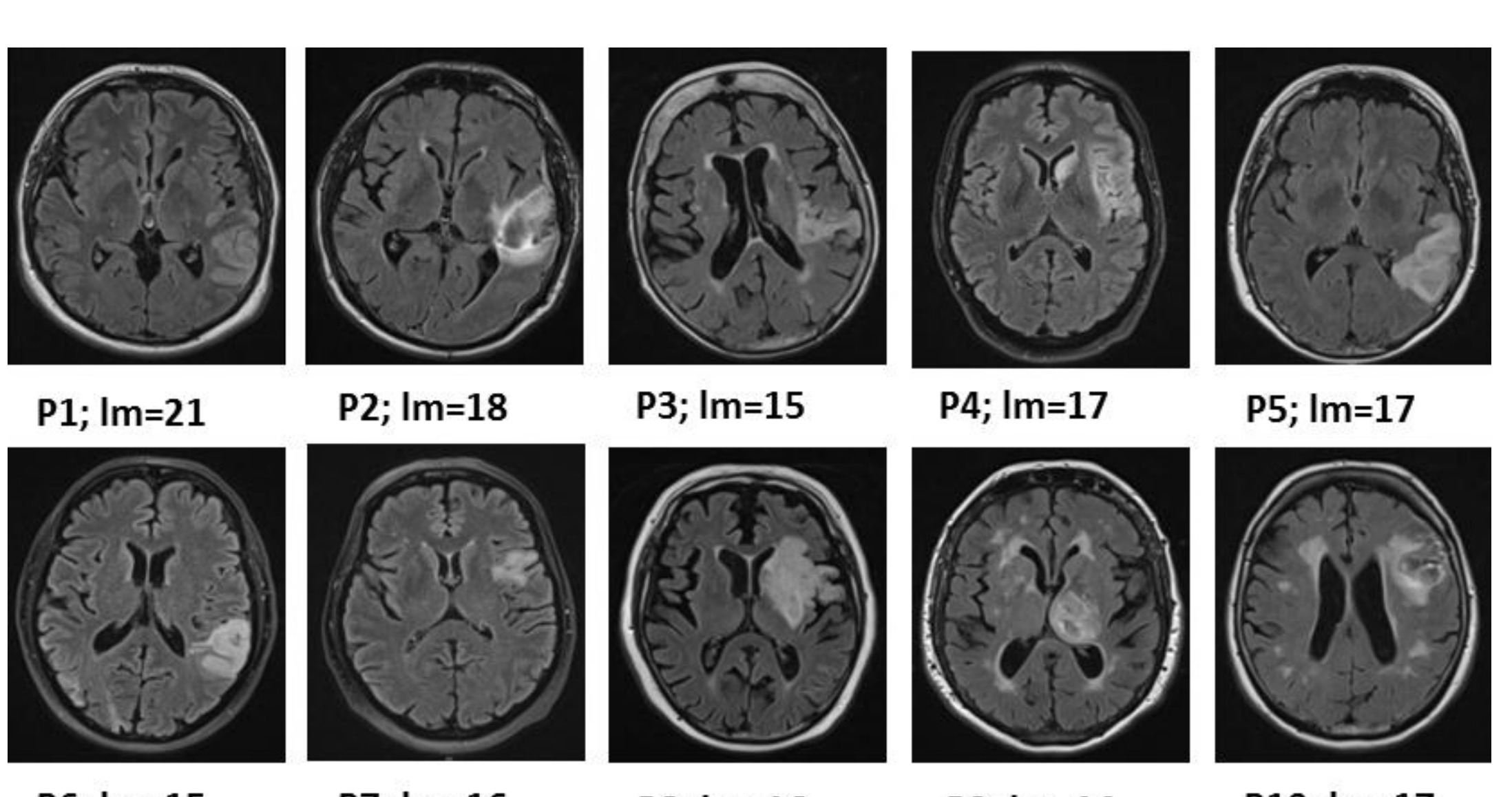

\section{Behavioural testing}

AAT (only patients)

PALPA (subtests phonological input)

\section{Neurophysiological testing}

Electroencephalogram (EEG) recorded through 23 electrodes

$\rightarrow$ international $10-20$ system

Phoneme discrimination based on 3 distinctive features (place, manner, voicing)

$\rightarrow 6$ oddball paradigms ( 3 distinctive features,

pre-attentive and attentive condition)

Word recognition based on RW - PW contras

$\rightarrow 1$ oddball paradigm (passive)

\section{Analysis (BrainVision Analyzer)}

Filter $0.5-30 \mathrm{~Hz} \rightarrow$ ICA $\rightarrow$ segmentation $\rightarrow$ artefact rejection $\rightarrow$ averaging stimuli $=$ ER

\section{References}

[1] Ilvonen, TM, et al, Stroke, 34, 1746-1753 (2003

[2] Nolfe, G, Cobianchi, A, Mossuto-Agatiello, L. \& Giaquinto, S., Eur I Neurol, 13, 377-384 (2006

[3] Aerts, A., van Mierlo, P., Hartsuiker, R., Santens, P. \& De Letter, M., Arch Sex Behav (under review

[3] Aerts, A., van Mierlo, P., Hartsuiker, R., Sa

[5] Möttö, et, R. \& Watkin, K.E., J Neurosi, 29(31), $9819-9825(2009)$

[6] van den Brink, D. Brown, C.M. \& Hagoot, P., JCOgn Neurosi, 13(7), 967-985 (2001

[7] Kawohl, W, Bunse, S., Willmes, K, Hoft, P., C. Bu, Neurosci, 13(7), 967,985 (2001) 282-289 (2010)

\section{RESULTS}

\section{Phoneme discrimination}

\begin{tabular}{c|ccc} 
& Place & Manner & Voicing \\
MMN & aphasia $=$ norm & aphasia $=$ norm & aphasia $<$ norm \\
P300 & aphasia $<$ norm & aphasia $<$ norm & aphasia < norm \\
\hline
\end{tabular}

MMN: more right-lateralized instead of left-lateralized = compensation mechanism ${ }^{[4]}$ ? Within the aphasic patients

$\rightarrow$ MMN: place > voicing; place $>$ manner; voicing = manner

$\rightarrow$ P300: place $>$ voicing; place $=$ manner; voicing $=$ manner

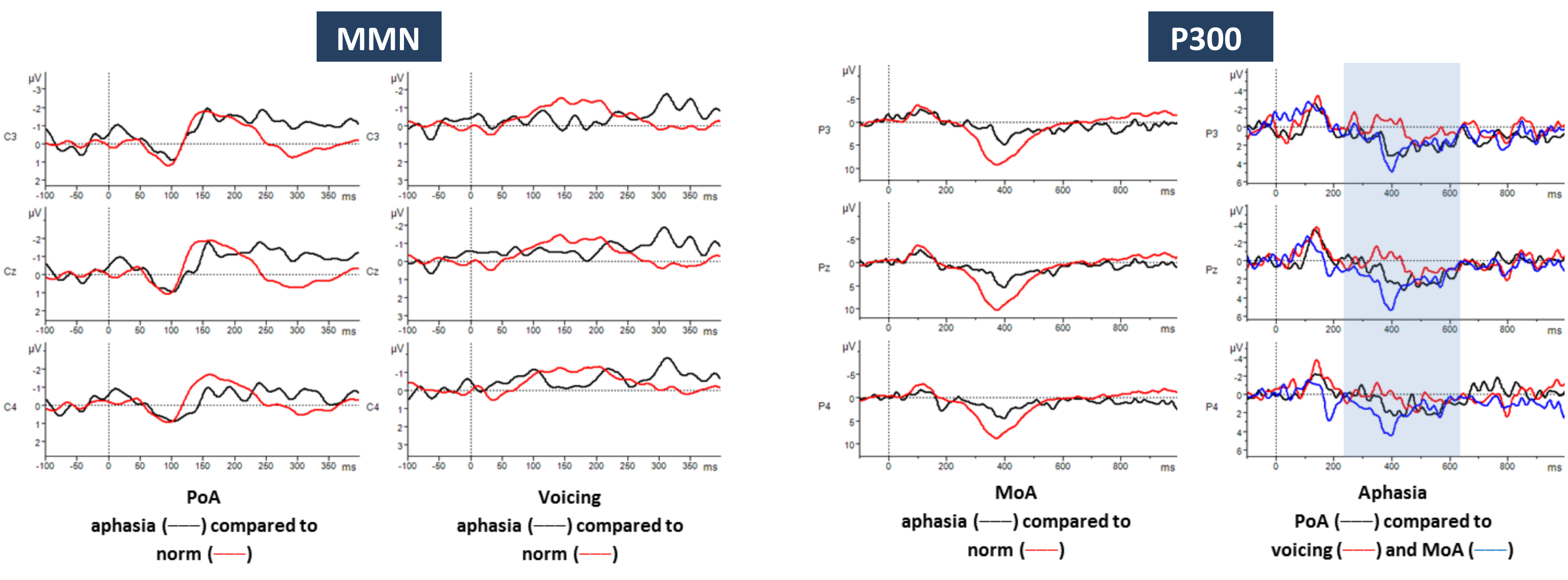

\section{Word recognition}

Aphasic patients

$\rightarrow$ intact pseudoword effect $=$ pseudowords $>$ real words (N100, P200, N400)

$\rightarrow$ P200: PW aphasic patients > PW norm group

BUT timing differences!!

$\rightarrow$ Aphasic patients are slower than the norm group during processing of pseudowords (N100, P200 and N400) and real words (P200)!
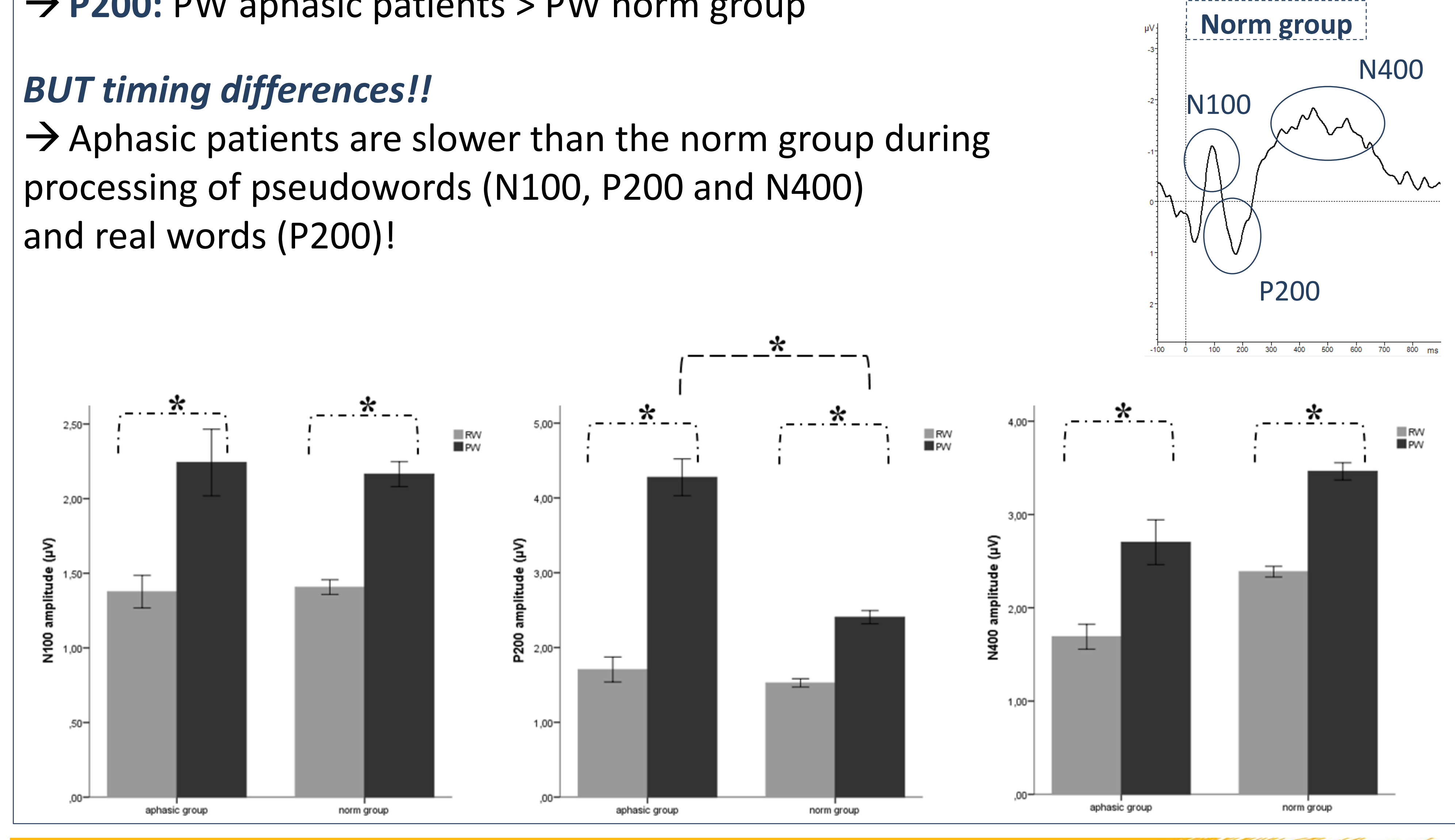

\section{DISCUSSION AND CONCLUSION}

\section{Phoneme discrimination}

Place of articulation less subject to neuronal loss?

$\rightarrow$ More spared than manner and voicing

$\rightarrow$ Better imprinted because of larger spectral differences and/or additional auditorymotor interface ${ }^{[5]}$ ?

Effect attention!!

$\rightarrow$ Pre-attentive $=$ only voicing diminished compared to norm

$\rightarrow$ Attentive $=$ all 3 distinctive features diminished compared to norm

$\rightarrow$ Neuronal resources for attention allocation supress neuronal activation dedicated to deviance detection?

\section{Word recognition}

Intact pseudoword effect in aphasic patients

$\rightarrow$ Detection of irregular phonological structure and difference in lexical status

$\rightarrow$ BUT more cognitive effort/less inhibition during processing of lexical properties (P200 个) ${ }^{[6]}$

$\rightarrow$ Less efficient information transfer (delay compared to the norm group) does not have a negative effect on lexical processing $\leftrightarrow$ disturbed semantic integration [7]

\section{Concluding remarks}

The paradigms seem to be sensitive enough for clinical, neurophysiological evaluation of phoneme discrimination and word recognition in acute aphasia HOWEVER

Be critical when using the attentive task ( $\mathrm{P} 300$ potential) because of other influencing cognitive factors!

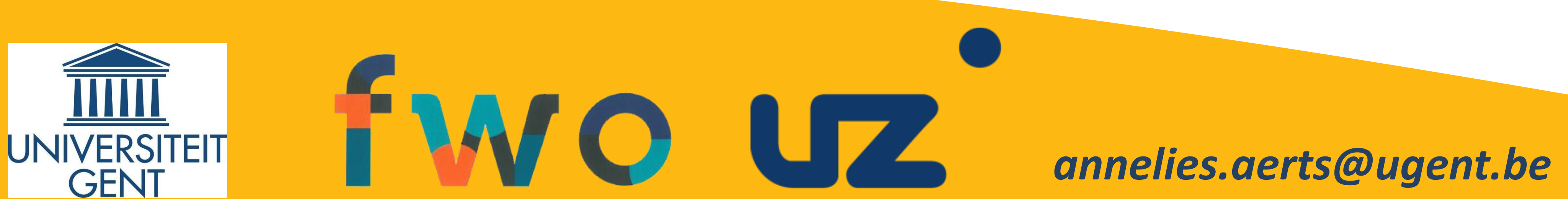

